Western University

Scholarship@Western

Obstetrics \& Gynaecology Publications

Obstetrics \& Gynaecology Department

6-1-2001

\title{
Amino acid concentrations in fluids from the bovine oviduct and uterus and in KSOM-based culture media.
}

Y M Elhassan

GWu

A C Leanez

RJ Tasca

AJ Watson

See next page for additional authors

Follow this and additional works at: https://ir.lib.uwo.ca/obsgynpub

Part of the Obstetrics and Gynecology Commons

Citation of this paper:

Elhassan, Y M; Wu, G; Leanez, A C; Tasca, R J; Watson, A J; and Westhusin, M E, "Amino acid concentrations in fluids from the bovine oviduct and uterus and in KSOM-based culture media." (2001). Obstetrics \& Gynaecology Publications. 60.

https://ir.lib.uwo.ca/obsgynpub/60 
Authors

Y M Elhassan, G Wu, A C Leanez, RJ Tasca, A J Watson, and M E Westhusin 


\title{
Levels of amino acids in bovine reproductive tract in comparison to culture medium supplemented with commercial amino acid stocks and various protein sources
}

\author{
YM Elhassan $^{1}$, AC Lean $\mathrm{ez}^{1}$, RJ Tasca ${ }^{2}, \mathrm{G} \mathrm{Wu}{ }^{3}$, AJ Watson ${ }^{4}, \mathrm{ME} \mathrm{Westhusin}^{1}$ \\ Departments of Veterinary Physiology and Pharmacology ${ }^{1}$ and Animal Science ${ }^{3}$, Texas A \& M \\ University, College Station, TX, 77843, USA; ${ }^{2}$ National Institute of Child Health and Human \\ Development, National Institutes of Health, Bethesda, MD, USA, ${ }^{4}$ Department of Obstetrics and \\ Gynaecology, University of Western Ontario, London, Canada.
}

\begin{abstract}
Aabstract
Oviductal (OF) and uterine (UF) fluids from slaughterhouse bovine reproductive tracts selected around the pre-implantation period of embryo development were analyzed for twenty-four amino acids. Amino acid concentrations in the OF and UF fluids were compared with those in a base culture medium (KSOM) supplemented with either FCS or Minimum Essential Medium amino acids (MEM-aa) in addition to PVA, FCS or BSA. Concentrations of 23 amino acids in UF were significantly $(\mathrm{P}<0.05)$ higher than those provided by KSOM supplemented with any of the supplements. Cystine was the only amino acid that was supplemented in comparable levels to those in UF. However, OF levels of CYS were higher than those in UF and supplemented KSOM. Compared to amino acid levels in OF, the supplementation of KSOM with either FCS or MEM-aa in addition to PVA, FCS or BSA resulted in significantly $(\mathrm{P}<0.05)$ lower levels of 15 , 13, 13 and 10 amino acids, respectively. The results showed that non-essential (NE) amino acids are the most abundant in both OF and UF, and compared to essential (E) amino acids, they were present in both fluids at ratios of 5:1 and 2:1, respectively. Glycine (14.1 mM), GLU (5.5 mM) and ALA $(3.7 \mathrm{mM})$ were present in high concentrations and comprised $72 \%$ of the free amino acid pool of OF. In the uterine fluid, concentrations of GLY (12.0 mM), GLU (4.2 mM), ALA (3.1 $\mathrm{mM})$ and SER $(2.7 \mathrm{mM})$ were the highest. Concentrations of all non-standard amino acids were significantly $(\mathrm{P}<0.05)$ higher in UF compared to KSOM supplemented with either FCS or FCS in addition to MEM-aa. In OF, only CIT and $\beta$-ALA were significantly $(\mathrm{P}<0.05)$ higher in concentrations compared to KSOM under similar supplementation. In conclusion, concentrations of most of the amino acids in the bovine reproductive tract were substantially higher than those supplemented in culture media commonly used for in vitro development of bovine preimplantation embryos. Adjustment of amino acid concentrations in the culture medium according to those found in the bovine reproductive tract may improve embryo development in vitro. Further culture studies are currently investigating this hypothesis.
\end{abstract}

Key words: Amino acids, Oviductal fluid, Uterine fluid, Culture medium 


\section{Introduction}

The key function of the oviduct in the reproductive process is to serve as the site of fertilization and early stages of embryonic development in addition to the transportation of cleavage-stage embryos to the uterus for implantation and further development. Some of the difficulties encountered in producing embryos of various species in vitro, such as the block stage, occur during the oviductal phase of development reflecting an important role of the oviduct during that phase. The importance of amino acids in embryo development in vitro has received much more attention in recent studies [1-4]. Essential and/or non-essential amino acids are common supplements in serum-supplemented or serum-free culture media used for mammalian embryo development in vitro. Their addition to the culture medium has been shown to be beneficial to bovine embryo development [5,6]. Apart from utilization in protein synthesis, amino acids play important roles as osmolytes [7,8], intracellular buffers [9], heavy metals chelators and energy sources $[10,11]$. They also function as precursors for versatile physiological regulators such as NO and polyamines [12]. Amino acids are used in culturing mammalian embryos in vitro, and in this respect are usually added to in vitro culture media as commercial stock solutions originally made to support growth of somatic cell in culture. Their requirement for optimum development of pre-implantation bovine embryos in culture has not yet been precisely determined. Levels of amino acids in the bovine reproductive tract during preimplantation embryonic development may provide a good reference for establishing optimum amino acid requirement for in vitro culture conditions. Little information is available on amino acid concentrations in bovine reproductive tract during early embryonic development. In this study we measured concentrations of amino acids in bovine oviductal and uterine fluids during pre-implantation embryonic development and compared them with those found in standard embryo culture media supplemented with commercial amino acid stocks and other protein sources.

\section{Materials and Methods}

Slaughterhouse bovine reproductive tracts with freshly ovulating follicles or very young corpora lutea were selected to approximate oviductal or uterine stages of embryonic development. Tracts were transported to the laboratory in ice-chilled plastic bags. Tissues surrounding oviducts and uterine horns were removed immediately upon arrival, and samples of oviductal fluid (OF) and uterine fluid (UF) were recovered from individual oviducts or uterine horns under aseptic conditions using Drummond pipettes (Drummon Scientific Companyd, PN). Following recovery, individual fluid samples were centrifuged at $13000 \mathrm{rpm}$ for 2-3 min and the supernatant was frozen in volumes of $20 \mathrm{ul}$ and stored at $-80{ }^{\circ} \mathrm{C}$ for later analysis of free amino acids. A modified simplex optimized medium (KSOM), a commonly used in vitro culture medium [13], was selected as base medium and supplemented with Minimun Essential Medium amino acid (MEM-aa) stocks or other protein sources for comparison of amino acid concentrations with those in the bovine reproductive tract. MEM-aa stocks comprised essential (MEM-E, Sigma 50x) and non-essential (MEM-NE, Sigma 100x) groups while supplemented protein sources included fetal calf serum (FCS) and bovine serum albumin (BSA). FCS, BSA, MEM-E and MEM-NE amino acids were added to the culture medium at $10 \%, 0.3 \%, 0.5 \mathrm{x}$ and 1.0x, respectively. Sample volumes of $100 \mathrm{ul}$ from each of the freshly 
prepared supplemented media were transferred into micro-centrifuge tubes and frozen at $-80{ }^{\circ} \mathrm{C}$ until analysis of individual amino acids was conducted.

\section{Amino acid analysis}

Analysis of amino acids by high-performance liquid chromatography was conducted according to the method described by $\mathrm{Wu}$ et. al. $[14,15]$ and included the standard essential and non-essential amino acids as well as a non-standard group comprised of $\beta$-alanine ( $\beta$-ALA), citrulline (CIT), ornithine (ORN) and taurine (TAU), ( Table 1). Values of UF and OF amino acid concentrations are respectively means of 8 and 10 samples taken from different animals, whereas samples for comparing amino acid levels between ovulation and non-ovulation sides were taken in pairs each from an animal using a total of 4-5 animals. The classification of amino acids into essential and nonessential used in this manuscript (Table 1) follows Sigma's classification of MEM amino acid stock solutions for cell culture (Sigma Chemical Inc., St. ois, Mo, 1998). The term non-standard is applied to $\beta$-ALA, CIT, ORN and TAU to identify them as a separate group. Since amino acid addition to the culture medium and their effect on embryo development is usually based on conventional classification of individual amino acids, the classification adopted in this study is used to facilitate comparing amino acids in OF and UF with those in the culture medium,

\section{Experiments}

Experiment 1: This experiment was designed to measure individual amino acids in bovine oviductal (OF) and uterine fluids (UF) and in KSOM culture medium supplemented with either FCS or MEM-E and NE amino acids in addition to PVA, BSA or FCS. MEM-E and NE amino acids were supplemented at a final concentrations of $0.5 \mathrm{x}$ and $1.0 \mathrm{x}$, while BSA and FCS were added at $0.3 \%$ and $10 \%$, respectively.

Experiment 2: In this experiment pairs of OF or UF samples were taken from each tract for individual amino acid analysis, with one sample being from the ovulation side and the other from the non ovulation one.

The purpose of the data produced from these experiments was to:

1) Determine individual essential, non-essential and non-standard amino acid levels and their relative abundance in bovine OF and UF during pre-implantation embryo development as a baseline for levels of amino acid to be added to culture media used for in vitro development of embryos.

2) Compare concentrations of essential, non-essential and non-standard amino acids in bovine OF and UF with those found in a commonly used in vitro culture medium supplemented with either FCS or MEM-aa in addition to PVA, BSA or FCS.

3) Investigate whether the side of ovulation affected essential, non-essential and non-standard amino acid levels in bovine OF or UF during pre-implantation embryo development.

\section{Statistical analysis}

Data were analyzed by ANOVA and Tukey's test for unbalanced data using the General Linear Model of SAS at a significant level of 5\% [16]. 


\section{Results}

The results of individual amino acid concentrations and their relative abundance in bovine oviductal and uterine fluids recovered from slaughterhouse reproductive tracts are shown in Table 2. The non-essential amino acid group was the most abundant in OF and comprised $82 \%$ of the total amino acids measured. In this group, GLY, GLU, ALA were present in high concentrations (14.1, 5,5 and $3.7 \mathrm{mM}$, respectively), and together represented $72 \%$ of the free amino acid pool in OF. Glycine alone represented $44 \%$ of total amino acid concentration. Concentrations of the essential amino acids in OF were relatively low and comprised less than $20 \%$ of total amino acids. In this group, CYS and THR were relatively high (1.4 and $0.8 \mathrm{mM}$, respectively) in OF compared to other essential amino acids (ranging from 0.1 to $0.5 \mathrm{mM}$ ). In the non-standard group, TAU and $\beta$-ALA showed the highest ( 0.46 and $0.14 \mathrm{mM}$ ) concentrations in $\mathrm{OF}$.

In the uterine fluid, non-essential amino acids represented $63 \%$ of the total concentration of amino acids, with GLY, GLU, ALA and SER showing the highest levels (12.0, 4.2, 3.1 and 2.7 $\mathrm{mM}$, respectively). The essential amino acids represented $29 \%$ of the total amino acid concentration in UF, with VAL, LEU, LYS, THR and ARG showing relatively high levels $(1.9,1.8,1.8,1.7$ and $1.4 \mathrm{mM}$, respectively). In the non-standard group, TAU showed the highest concentration $(3.4 \mathrm{mM})$ in UF.

The results of the comparison between individual amino acid levels in OF and UF and those in KSOM culture medium supplemented with various protein sources is shown in table 3 . Concentrations of the essential amino acids HIS, THR, TRP, VAL, PHE, ILE, LEU and CYS were significantly lower $(\mathrm{P}<0.05)$ in KSOM supplemented with FCS alone compared to OF, whereas concentrations of ARG, TYR, MET and LYS were comparable (P>0.05) between the two. Further addition of MEM-aa to the FCS-supplemented KSOM culture medium brought concentrations of the essential amino acids HIS, VAL, PHE, ILE and LEU to levels comparable (P>0.05) to those present in OF. However, concentration of THR, TRP and CYS in OF remained significantly lower $(\mathrm{P}<0.05$ in FCS-supplemented KSOM compared to OF following the addition of MEM-aa. When KSOM culture medium was supplemented with MEM-aa in addition to either BSA or PVA, concentration of all essential amino acids, with the exception of THR, TRP, ILE and CYS, were comparable $(\mathrm{P}>0.05)$ to those found in $\mathrm{OF}$. The latter were significantly lower $(\mathrm{P}<0.05)$ compared to OF. Concentration of the non-essential amino acids ASP, GLU, GLY, ALA and PRO in KSOM culture medium supplemented with either FCS, or MEM-aa in addition to PVA, BSA or FCS were significantly lower $(\mathrm{P}<0.05)$ than their levels in $\mathrm{OF}$. Concentration of the non-essential amino acids ASN, SER and GLN in KSOM culture medium supplemented with each of the protein supplements were comparable $(\mathrm{P}>0.05)$ to their levels in $\mathrm{OF}$.

Comparison of non-standard amino acid concentrations in OF, UF and KSOM culture did not include KSOM supplementation with either MEM-aa or BSA, because the former is free of these amino acids and the latter did not show detectable levels. In this group, concentration of CIT and $\beta$-ALA were significantly lower $(\mathrm{P}<0.05)$ in FCS-Supplemented KSOM compared to OF $(0.01$ and 0.01 vs 0.1 and 0.1 , respectively). In UF, concentrations of CIT, $\beta$-ALA, TAU and ORN were significantly higher $(\mathrm{P}<0.05)$ than their concentrations in FCS-supplemented KSOM $(0.1,0.1,3.4$ and 0.2 vs $0.01,0.01,0.01$ and 0.02 , respectively).

With the exception of CYS, concentrations of all amino acids in KSOM culture medium supplemented with either FCS or MEM-aa in addition to PVA, FCS or BSA were significantly lower $(\mathrm{P}<0.05)$ compared to UF (Table 3). Cystine concentrations in supplemented KSOM were comparable $(\mathrm{P}>0.05)$ to those in UF. 
The results of amino acid concentrations in OF and UF samples taken from the ovulation or non-ovulation sides are shown in table 4 . There was no difference $(\mathrm{P}>0.05)$ in amino acid concentrations between OF samples taken from the ovulation or non-ovulation sides in all groups of amino acids. Uterine fluid samples showed no difference $(\mathrm{P}>0.05)$ in amino acid levels between ovulation and non-ovulation sides except for the essential amino acid ARG and the non-essential amino acid ASP which were present in significantly higher $(\mathrm{P}<0.05)$ levels in the side of ovulation than the non-ovulation side.

\section{Discussion}

Few attempts were made decades ago to measure levels of amino acids in bovine oviductal and/or uterine fluids [17-19] using different techniques of fluid collection and experimental materials including live animals and reproductive tracts. Moor and Bondioli [2] recently reported amino acid concentrations in obstructed or surgically ligated oviducts of live animals over a minimum period of one estrous cycle for fluid collection. Fluids collected by such techniques are not reflective of the natural in vivo conditions, and are probably altered by induced inflammatory or pathological conditions, affecting levels of individual amino acids measured. This may possibly explain in part the wide variations observed in results obtained from different studies [2, 17-19]. In the previous studies data on amino acids were incomplete probably due to the sensitivity of the analysis or the technical difficulties involved. Moreover, none of the previous studies has compared concentrations of amino acids in the bovine reproductive tract with those provided in culture media used for in vitro development of embryos. In this study we quantified OF and UF amino acids around pre-implantation embryo development using freshly excised tracts, and compared them with those available in a culture medium commonly used for in vitro development of bovine embryos.

The results presented in this study showed that non-essential amino acids are more abundant in both OF and UF than essential amino acids. Interestingly, the ratio between non-essential and essential amino acids was different between OF and UF (5:1 vs 2:1, respectively) due to elevated levels of essential amino acids in UF. Ratios of amino acids are known to be critical for protein synthesis in living cells, and the current observation is worth investigating whether the ratio rather than the absolute value of amino acid levels is more important for embryo development. This notion is supported by studies reporting improved embryo development with supplementation of nonessential amino acids alone [5,6] or in combination with essential amino acids at a ratio of 2:1 [5] during the first 2-3 embryonic cleavages. Other studies indicated that some amino acids are present in very low concentration in ovine OF suggesting that they might be embryotoxic at normal plasma levels [20].

Glycine, GLU, and ALA were found in highest levels (>3 mM) in OF while GLY, GLU, ALA and TAU were the most abundant in UF. Other studies [19, 21] also found GLY, GLU and ALA among the most abundant amino acids measured in the bovine reproductive tract. Moor and Bondioli [2] found GLY and ALA highest in concentration but unable to detect a number of amino acids including ASP, ASN, GLN, HIS, TYR, CYS, ILE, LEU, PHE and TRP. However, concentrations of amino acids reported in these studies were lower than concentrations reported in this study. This difference is probably due to concentration variations associated with the stage of the cycle, dilution effect of flushing fluids or pathological conditions precipitated either by tubal ligation or introduction of catheters during sampling.

In this study, levels of GLY were strikingly high both in OF (14.1 mM) and UF (12.0mM) comprising the highest percent of the free amino acid pool in each of these fluids (44 and 27\%, 
respectively). This high concentration of GLY suggests an unusual role(s) for this amino acid in the reproductive tract. Similar findings of high GLY levels were previously reported in bovine [2], ovine [22,23], rabbit [24], mare [25] and the mouse [26]. Leese and Gray, [27] reported that the secretion rate of GLY into the rabbit oviduct lumen was the highest of the amino acids and it comprised $70 \%$ of the amino acid pool in blastocysts. Aside from being a building block for protein synthesis, GLY has been shown to be one of the most effective organic osmoregulators for the mouse embryo [28-30]. It is possible that GLY plays similar role during preimplantation development of bovine embryos, protecting them from the high oviductal and uterine osmolarity (350-370 mOsM). Interestingly, GLY is the most abundant amino acid in the fetus throughout gestation [31].

Cystine and $\beta$-alanine were the only two amino acids that were present in higher levels in OF than in UF. This possibly suggests special roles for these amino acids during oviductal phase of embryo development. Cystine, by providing the reactive thiol group, is a keystone to a number of functions fulfilled by glutathione. One of these functions is the detoxification of exogenous and endogenous reactive electrophiles and toxic oxygen metabolites [32]. In this regard, CYS is possibly very effective in the oviduct compared to the uterus where TAU, another antioxidant, is present at higher levels than in the oviduct. $\beta$-ALA is one of several effective organic osmoprotectants in addition to GLY [29,30], and is possibly needed at these levels in the oviduct to guard, with GLY and possibly other substrates, the developing embryos from harmful effects of high osmolarity. Uterine osmolarity is as high as that of the oviduct, and GLY and TAU probably regulate osmolarity more effectively in the uterus in contrast to the oviduct. However, these observations need to be tested experimentally.

The results of the comparison between amino acids concentrations in OF and UF and those in KSOM culture medium showed that none of KSOM supplements brought any of the essential amino acids, except CYS, to levels comparable to those in UF. In the case of OF, each of the various supplements added to KSOM raised the concentrations of some amino acids to oviductal levels. However, concentrations of some essential (THR, TRP, CYS and ILE), non-essential (ASP, GLU, GLY, ALA and PRO) and non-standard (CIT and $\beta$-ALA) amino acids remained at significantly lower levels compared to OF levels irrespective of the supplement added. Fetal calf serum (FCS) as a sole supplement to KSOM failed to bring additional 4 essential amino acids (VAL, PHE, ILE and LEU) to levels comparable to those in OF. As a result, a total of 8 amino acids were relatively deficient in FCS-supplemented KSOM. Compared to the supplementation of FCS alone, the addition of MEM-aa to KSOM supplemented with PVA or BSA reduced the number of essential amino acids with levels lower than OF levels to 4 (THR, TRP, CYS and ILE). When MEM-aa were added to KSOM supplemented with FCS, the number of deficient essential amino acids was 3 (THR, TRP and CYS). These findings indicate that 10\% FCS, as the only supplement, is the poorest source of amino acids in term of providing levels comparable to those in OF and UF. On the other hand, supplementation of KSOM with MEM-aa was better than FCS but the number of amino acids deficient was still more than half of the total amino acids measured. Additional supplementation of KSOM with FCS in the presence of MEM-aa brought level of one additional essential amino acid (ILE) to its OF level while that with BSA contributed nothing.

In conclusion, the concentrations of 23 out of 24 amino acids measured in the bovine uterine fluid around the pre-implantation period of embryo development were significantly higher than those found in a standard culture medium (KSOM) supplemented with either FCS or MEM-aa in addition to PVA, BSA or FCS. In the case of OF, concentrations of 15, 13, 13 and 10 amino acids were significantly higher than those in KSOM supplemented with either FCS or MEM-aa in 
addition to PVA, BSA or FCS, respectively. Studies in sheep [33] demonstrated that embryos cultured in a defined medium supplemented with amino acids at ovine oviductal fluid levels were able to develop into conceptuses at relatively high rates. We hypothesize that two-step in vitro culture of preimplantation bovine embryos in a defined culture medium supplemented with amino acids at OF followed by UF levels is expected to improve development, quality and viability of bovine embryos. This hypothesis is based on the rationale that measured bovine reproductive tract levels of amino acids are indicative of optimum requirement for bovine embryo development in vitro. Currently we are conducting culture experiments on in vitro development of preimplantation bovine embryos using culture media supplemented with amino acids at oviductal and uterine levels to test this hypothesis.

\section{References}

1. Takahashi Y, First NL, 1992. In vitro development of bovine one-cell embryos: influence of glucose, lactate, pyruvate, amino acids and vitamins. Theriogenology 37:963-978.

2. Moore K, Bondioli K. Glycine and alanine supplementation of culture medium enhances development of in vitro matured and fertilized cattle embryos. Biol Reprod 1993; 48:833840.

3. Rosenkrans CF Jr., First NL. Effect of free amino acids and vitamins on cleavage and developmental rate of bovine zygotes in vitro. J An Sci 1993; 72:434-437.

4. Gardner DK, Lane M, Spitzer A, Batt PA. Enhanced rates of cleavage and development for sheep zygotes cultured to the blastocyst stage in vitro in the absence of serum and somatic cells: amino acids, vitamins, and culturing embryos in groups stimulate development. Biol Reprod 1994; 50:390-400.

5. Liu Z, Foote RH. Effects of amino acids on the development of in-vitro matured/in-vitro fertilized bovine embryos in a simple protein-free medium. Hum Reprod 1995; 10: 29852991.

6. Pinyopummintr T, Bavister BD. Effects of amino acids on development in vitro of cleavagestage bovine embryos into blastocysts. Reprod Fertil Devel 1996; 8:835-841.

7. Anbari K, Schultz RM. Effect of sodium and betaine in culture media on development and relative rates of protein synthesis in preimplantation mouse embryo in vitro. Mol Reprod Devel 1993; 35:24-28.

8. Biggers JD, Lawitts JA, Lechene CP. The protective action of betaine on the deleterious effects of $\mathrm{NaCl}$ on preimplantation mouse embryos in vitro. Mol. Reprod. Devel 1993; 34:380-390.

9. Bavister BD, McKiernan SH. Regulation of hamster embryo development in vitro by amino acids. In: Baviaster, B.D. (ed), Preimplantation embryo development, Springer-Verlag, New York, 1993; pp.57-72.

10. Rieger D. Relationship between energy metabolism and development of early mammalian embryos. Theriogenology 1992; 37:75-93.

11. Bavister BD. Culture of preimplantation embryos: facts and artifacts. Hum. Reprod. Upd., 1995; 1:91-148.

12. Wu G, Morris SM Jr. Arginine metabolism: nitric oxide and beyond. Biochem J 1998; 336:1 17.

13. Lawitts JA, Biggers JD. Culture of preimplantation embryos. In: Wassarman PM, DePamphilis ML (eds.), Methods in Enzymology. Vol. 225 (Guide to Techniques in Mouse Development). San Diego, CA: Academic Press; 1993: 153-164. 
14. Wu G, Bazer FW, Tuo W, Flynn SP. Unusual abundance of arginine and ornithine in porcine allantois fluid. Biol Reprod 1996; 54:1261-1265.

15. Wu G, Davis PK, Flynn NE, Knabe DA, Davidson JT. Endogenous synthesis of arginine plays an important role in maintaining arginine homeostasis in postweaning growing pigs. J Nutr 1997; 127: 2342-2349.

16. SAS. SAS/STAT User's Guide, version 6.12 edition. Cary, NC: Statistical Analysis System Institute, Inc.; 1998.

17. Fahning ML, Scultz RH, Graham EF. The free amino acid content of uterine fluids and blood serum in the cow. J Reprod Fert 1967; 13:229-236.

18. Loe WC, Roussel JD, Patrick TE. Protein and amino acid content of uterine and oviduct fluid of dairy heifers. J Dairy Sci 1970; 53: 662, Abstr.

19. Stanke DF, Sikes JD, DeYoung DW, Tumbleson ME. Proteins and amino acids in bovine oviductal fluid. J Reprod Fert 1974; 38:493-496.

20. Nancarrow CD, Hill JL. Co-culture, oviduct secretion and the function of oviduct-specific glycoproteins. Cell Biol Intern 1994; 18:1105-1114.

21. Iritani A, Nishikawa Y, Gomes WR, VanDemark NL. Secretion rates and chemical composition of oviduct and uterine fluids in rabbits. J Anim Sci 1971; 33:829-835.

22. Hill JL, Wade MG, Nancarrow DL, Kelleher DL, Boland MP. Influence of ovine oviductal amino acid concentrations and an ovine oestrus-associated glycoprotein on development and viability of bovine embryo. Mol Reprod Develop 1997; 47:164-169.

23. Moses DF, Matkovic M, Cabrera Fisher E, Martinez AG. Amino acid contents on sheep oviductal and uterine fluids. Theriogenology 19971; 47: 336.

24. Miler JGO, Schultz GA. Amino acid contents of preimplantation rabbit embryos and fluids of the reproductive tract. Biol Reprod 1987; 36:125-129.

25. Engle CC, Foley CW, Plotka ED, Witherspoon DM. Free amino acids and protein concentrations in reproductive tract fluids of the mare. Theriogenology 1984; 21:919-930.

26. Menezo Y, Hamidi J, Khatchadourian C, Guillaud J, Nardon C. Culture of the mouse egg in the prepuberal mouse oviduct. In: Proceedings of the $11^{\text {th }}$ International Congress of Anim Reprod AI; Dublin, Ireland. 1988; Abstract 477.

27. Leese HJ, Gary, SM. Vascular perfusion: a novel means of studying oviduct function. Am J Physiol 1985; 248: E624-E632.

28. Van Winkle LJ, Haghighat N, Campione AL. Glycine protects preimplantation mouse conceptuses from a deterimental effect on development of the inorganic ions in oviductal fluid. J Exp Zoo 1990; 253:215-219.

29. Dawson KM, Baltz JM. Organic osmolytes and embryos: substrates of the Gly and beta transport systems protect mouse zygotes against the effects of raised osmolarity. Biol Reprod 1997; 56:1550-1558.

30. Dawson KM, Collins JL, Baltz JM. Osmolarity-dependent glycine accumulation indicates a role for glycine as an organic osmolyte in early preimplantation mouse embryos. Biol of Reprod 1998; 59:225-32.

31. Wu G, Ott TL, Knabe DA, Bazer FW. Amino acid composition of the fetal pig. J Nutr 1999; 129:1031-1038.

32. DeLeve LD, Kaplowitz N. Glutathione metabolism and its role in hepatotoxicity. Pharmac Ther 1991; 52:287-305. 
33. Walker SK, Hill JL, Kleemann DO, Nancarrow, CD. Development of ovine embryos in synthetic oviductal fluid containing amino acid at oviductal fluid concentrations. Biol Reprod 1996; 55:703-708. 
Table 1. Names and codes of essential, non-essential and non-standard amino acids. *

\begin{tabular}{|c|c|c|c|c|c|c|c|c|}
\hline Code & Name & Class & Code & Name & Class & Code & Name & Class \\
\hline HIS & Histidine & $\mathrm{E}$ & ILE & Isoleucine & $\mathrm{E}$ & GLN & Glutamine & $\mathrm{NE}$ \\
\hline THR & Threonine & $\mathrm{E}$ & LEU & Leucine & $\mathrm{E}$ & GLY & Glycine & $\mathrm{NE}$ \\
\hline ARG & Arginine & $\mathrm{E}$ & LYS & Lysine & $\mathrm{E}$ & $\mathrm{AlA}$ & Alanine & $\mathrm{NE}$ \\
\hline TYR & Tyrosine & $\mathrm{E}$ & CYS & Cystine & $\mathrm{E}$ & PRO & Proline & $\mathrm{NE}$ \\
\hline TRP & Tryptophan & $\mathrm{E}$ & ASP & Aspartic acid & $\mathrm{NE}$ & CIT & Citrulline & NS \\
\hline MET & Methionine & $\mathrm{E}$ & GLU & Glutamic acid & $\mathrm{NE}$ & $\beta$ ALA & $\beta$ Alanine & NS \\
\hline VAL & Valine & $\mathrm{E}$ & ASN & Asparagine & $\mathrm{NE}$ & TAU & Taurine & NS \\
\hline PHE & Phenylalanine & $\mathrm{E}$ & SER & Serine & $\mathrm{NE}$ & ORN & Ornithine & NS \\
\hline
\end{tabular}

* According to Sigma's classification of MEM-aa into essential (E) and non-essential (NE) amino acids (Sigma Chemical Inc., St. ois, Mo, 1998); NS=non-standard amino acids. 
Table 2. Levels of amino acids and their relative abundance in bovine oviductal and uterine fluids.

\begin{tabular}{lrrrr}
\hline Amino Acid & OF-aa & $\begin{array}{c}\text { Percent in } \\
\text { OF-aa }\end{array}$ & UF-aa & $\begin{array}{c}\text { Percent in } \\
\text { UF-aa }\end{array}$ \\
(aa) & $(\mathrm{mM})$ & $(\%)$ & $(\mathrm{mM})$ & \multicolumn{1}{c}{$\%)$} \\
\hline HIS & $0.17 \pm 0.01$ & $0.55 \pm 0.00$ & $0.59 \pm 0.05$ & $1.31 \pm 0.00$ \\
THR & $0.83 \pm 0.06$ & $2.62 \pm 0.00$ & $1.69 \pm 0.15$ & $3.78 \pm 0.00$ \\
ARG & $0.28 \pm 0.02$ & $0.88 \pm 0.00$ & $1.4 \pm 0.12$ & $3.26 \pm 0.00$ \\
TYR & $0.18 \pm 0.01$ & $0.56 \pm 0.00$ & $0.63 \pm 0.06$ & $1.4 \pm 0.00$ \\
TRP & $0.15 \pm 0.01$ & $0.48 \pm 0.00$ & $0.28 \pm 0.01$ & $0.65 \pm 0.00$ \\
MET & $0.1 \pm 0.01$ & $0.31 \pm 0.00$ & $0.55 \pm 0.05$ & $1.23 \pm 0.00$ \\
VAL & $0.49 \pm 0.05$ & $1.55 \pm 0.00$ & $1.85 \pm 0.14$ & $4.15 \pm 0.00$ \\
PHE & $0.22 \pm 0.02$ & $0.68 \pm 0.00$ & $0.7 \pm 0.05$ & $1.56 \pm 0.00$ \\
ILE & $0.31 \pm 0.01$ & $0.98 \pm 0.00$ & $0.87 \pm 0.06$ & $1.96 \pm 0.00$ \\
LEU & $0.48 \pm 0.04$ & $1.5 \pm 0.00$ & $1.81 \pm 0.13$ & $4.08 \pm 0.00$ \\
LYS & $0.39 \pm 0.03$ & $1.22 \pm 0.00$ & $1.79 \pm 0.16$ & $3.99 \pm 0.00$ \\
CYS & $1.35 \pm 0.19$ & $4.14 \pm 0.01$ & $0.55 \pm 0.07$ & $1.34 \pm 0.00$ \\
ASP & $0.77 \pm 0.07$ & $2.45 \pm 0.00$ & $1.75 \pm 0.16$ & $3.91 \pm 0.00$ \\
GLU & $5.47 \pm 0.48$ & $17.23 \pm 0.01$ & $4.23 \pm 0.18$ & $9.67 \pm 0.00$ \\
ASN & $0.11 \pm 0.01$ & $0.33 \pm 0.00$ & $0.4 \pm 0.06$ & $0.86 \pm 0.00$ \\
SER & $0.62 \pm 0.02$ & $1.99 \pm 0.00$ & $2.68 \pm 0.22$ & $5.97 \pm 0.00$ \\
GLN & $0.94 \pm 0.13$ & $2.96 \pm 0.00$ & $1.84 \pm 0.13$ & $4.14 \pm 0.00$ \\
GLY & $14.06 \pm 1.21$ & $43.98 \pm 0.03$ & $11.99 \pm 1.13$ & $26.77 \pm 0.01$ \\
ALA & $3.68 \pm 0.33$ & $11.43 \pm 0.01$ & $3.13 \pm 0.24$ & $7.04 \pm 0.00$ \\
PRO & $0.57 \pm 0.07$ & $1.81 \pm 0.00$ & $1.88 \pm 0.11$ & $4.29 \pm 0.00$ \\
CIT & $0.09 \pm 0.01$ & $0.29 \pm 0.00$ & $0.09 \pm 0.01$ & $0.22 \pm 0.00$ \\
$\beta$-ALA & $0.14 \pm 0.01$ & $0.43 \pm 0.00$ & $0.08 \pm 0.01$ & $0.2 \pm 0.00$ \\
TAU & $0.46 \pm 0.07$ & $1.45 \pm 0.00$ & $3.43 \pm 0.48$ & $7.89 \pm 0.01$ \\
ORN & $0.06 \pm 0.01$ & $0.19 \pm 0.00$ & $0.15 \pm 0.02$ & $0.34 \pm 0.00$ \\
\hline
\end{tabular}

Data are means \pm SEM, $\mathrm{n}=10$ and 8 for $\mathrm{OF}$-aa and UF-aa, respectively. OF-aa=oviductal fluid amino acids; UF-aa=uterine fluid amino acids. 
Table 3. Levels of amino acids in oviductal and uterine fluids in comparison to KSOM culture medium supplemented with various protein sources

\begin{tabular}{|c|c|c|c|c|c|c|}
\hline $\begin{array}{l}\text { Amino } \\
\text { acid } \\
\text { (aa) }\end{array}$ & OF-aa & UF-aa & $\mathrm{K}+\mathrm{FCS}$ & $\begin{array}{l}\text { K+FCS+ } \\
\text { MEM-aa } \\
(\mathrm{mM})\end{array}$ & $\begin{array}{l}\text { K+BSA+ } \\
\text { MEM-aa } \\
(\mathrm{mM})\end{array}$ & $\begin{array}{l}\text { K-PVA + } \\
\text { MEM-aa } \\
(\mathrm{mM})\end{array}$ \\
\hline HIS & $0.17 \pm 0.01^{b}$ & $0.59 \pm 0.05^{\mathrm{a}}$ & $0.01 \pm 0.00^{\mathrm{c}}$ & $0.10 \pm 0.01^{b c}$ & $0.08 \pm 0.08^{b c}$ & $0.07 \pm 0.01^{b c}$ \\
\hline THR & $0.83 \pm 0.06^{\mathrm{b}}$ & $1.69 \pm 0.15^{\mathrm{a}}$ & $0.02 \pm 0.00^{\mathrm{c}}$ & $0.22 \pm 0.02^{c}$ & $0.20 \pm 0.20^{c}$ & $0.18 \pm 0.01^{\mathrm{c}}$ \\
\hline ARG & $0.28 \pm 0.02^{b}$ & $1.40 \pm 0.12^{\mathrm{a}}$ & $0.01 \pm 0.00^{b}$ & $0.30 \pm 0.02^{b}$ & $0.29 \pm 0.29^{b}$ & $0.28 \pm 0.01^{b}$ \\
\hline TYR & $0.18 \pm 0.01^{b c}$ & $0.63 \pm 0.06^{\mathrm{a}}$ & $0.02 \pm 0.00^{\mathrm{c}}$ & $0.20 \pm 0.02^{b}$ & $0.19 \pm 0.20^{b}$ & $0.17 \pm 0.01^{b c}$ \\
\hline TRP & $0.15 \pm 0.01^{\mathrm{b}}$ & $0.28 \pm 0.01^{\mathrm{a}}$ & $0.01 \pm 0.00^{\mathrm{c}}$ & $0.02 \pm 0.00^{\mathrm{c}}$ & $0.02 \pm 0.03^{\mathrm{c}}$ & $0.02 \pm 0.00^{c}$ \\
\hline MET & $0.10 \pm 0.01^{b}$ & $0.55 \pm 0.05^{\mathrm{a}}$ & $0.00 \pm 0.00^{\mathrm{b}}$ & $0.05 \pm 0.01^{b}$ & $0.04 \pm 0.05^{b}$ & $0.04 \pm 0.00^{b}$ \\
\hline VAL & $0.49 \pm 0.05^{b}$ & $1.85 \pm 0.14^{\mathrm{a}}$ & $0.04 \pm 0.01^{\mathrm{c}}$ & $0.24 \pm 0.02^{b c}$ & $0.20 \pm 0.20^{b c}$ & $0.19 \pm 0.01^{b c}$ \\
\hline PHE & $0.22 \pm 0.02^{b}$ & $0.70 \pm 0.05^{\mathrm{a}}$ & $0.01 \pm 0.00^{\mathrm{c}}$ & $0.11 \pm 0.01^{b c}$ & $0.10 \pm 0.10^{b c}$ & $0.09 \pm 0.01^{b c}$ \\
\hline ILE & $0.31 \pm 0.01^{b}$ & $0.87 \pm 0.06^{\mathrm{a}}$ & $0.08 \pm 0.02^{c}$ & $0.21 \pm 0.04^{b c}$ & $0.10 \pm 0.11^{\mathrm{c}}$ & $0.11 \pm 0.01^{\mathrm{c}}$ \\
\hline LEU & $0.48 \pm 0.04^{b}$ & $1.81 \pm 0.13^{\mathrm{a}}$ & $0.03 \pm 0.00^{c}$ & $0.23 \pm 0.02^{b c}$ & $0.20 \pm 0.20^{b c}$ & $0.19 \pm 0.01^{b c}$ \\
\hline LYS & $0.39 \pm 0.03^{b}$ & $1.79 \pm 0.16^{\mathrm{a}}$ & $0.02 \pm 0.01^{b}$ & $0.22 \pm 0.01^{b}$ & $0.20 \pm 0.20^{\mathrm{b}}$ & $0.19 \pm 0.00^{b}$ \\
\hline CYS & $1.35 \pm 0.19^{\mathrm{a}}$ & $0.55 \pm 0.07^{b}$ & $0.01 \pm 0.00^{\mathrm{b}}$ & $0.12 \pm 0.01^{b}$ & $0.10 \pm 0.11^{b}$ & $0.09 \pm 0.01^{b}$ \\
\hline ASP & $0.77 \pm 0.07^{b}$ & $1.75 \pm 0.16^{\mathrm{a}}$ & $0.01 \pm 0.00^{\mathrm{c}}$ & $0.12 \pm 0.02^{\mathrm{c}}$ & $0.10 \pm 0.11^{\mathrm{c}}$ & $0.09 \pm 0.01^{\mathrm{c}}$ \\
\hline GLU & $5.47 \pm 0.48^{\mathrm{a}}$ & $4.23 \pm 0.18^{\mathrm{a}}$ & $0.09 \pm 0.01^{b}$ & $0.24 \pm 0.06^{b}$ & $0.12 \pm 0.13^{b}$ & $0.11 \pm 0.00^{b}$ \\
\hline ASN & $0.11 \pm 0.01^{b}$ & $0.40 \pm 0.06^{\mathrm{a}}$ & $0.01 \pm 0.00^{b}$ & $0.09 \pm 0.00^{b}$ & $0.09 \pm 0.09^{b}$ & $0.08 \pm 0.00^{b}$ \\
\hline SER & $0.62 \pm 0.02^{b}$ & $2.68 \pm 0.22^{\mathrm{a}}$ & $0.03 \pm 0.01^{b}$ & $0.12 \pm 0.01^{b}$ & $0.10 \pm 0.12^{b}$ & $0.10 \pm 0.00^{b}$ \\
\hline GLN & $0.94 \pm 0.13^{b}$ & $1.84 \pm 0.13^{\mathrm{a}}$ & $1.04 \pm 0.03^{b}$ & $1.05 \pm 0.08^{b}$ & $1.05 \pm 1.07^{b}$ & $0.97 \pm 0.03^{b}$ \\
\hline GLY & $14.06 \pm 1.21^{\mathrm{a}}$ & $11.99 \pm 1.13^{\mathrm{a}}$ & $0.13 \pm 0.03^{b}$ & $0.36 \pm 0.01^{b}$ & $0.12 \pm 0.10^{b}$ & $0.11 \pm 0.01^{b}$ \\
\hline ALA & $3.68 \pm 0.33^{\mathrm{a}}$ & $3.13 \pm 0.24^{\mathrm{a}}$ & $0.12 \pm 0.01^{b}$ & $0.26 \pm 0.04^{b}$ & $0.13 \pm 0.13^{b}$ & $0.11 \pm 0.01^{b}$ \\
\hline PRO & $0.57 \pm 0.07^{b}$ & $1.88 \pm 0.11^{\mathrm{a}}$ & $0.04 \pm 0.01^{\mathrm{c}}$ & $0.13 \pm 0.01^{\mathrm{c}}$ & $0.11 \pm 0.09^{c}$ & $0.10 \pm 0.00^{\mathrm{c}}$ \\
\hline CIT & $0.09 \pm 0.01^{\mathrm{a}}$ & $0.09 \pm 0.01^{\mathrm{a}}$ & $0.01 \pm 0.00^{\mathrm{b}}$ & $0.01 \pm 0.00^{b}$ & N/D & $\mathrm{N} / \mathrm{S}$ \\
\hline$\beta$-ALA & $0.14 \pm 0.01^{\mathrm{a}}$ & $0.08 \pm 0.01^{b}$ & $0.01 \pm 0.00^{\mathrm{c}}$ & $0.01 \pm 0.00^{\mathrm{c}}$ & N/D & $\mathrm{N} / \mathrm{S}$ \\
\hline TAU & $0.46 \pm 0.07^{b}$ & $3.43 \pm 0.48^{\mathrm{a}}$ & $0.01 \pm 0.01^{b}$ & $0.02 \pm 0.00^{b}$ & N/D & $\mathrm{N} / \mathrm{S}$ \\
\hline ORN & $0.06 \pm 0.01^{b}$ & $0.15 \pm 0.02^{\mathrm{a}}$ & $0.02 \pm 0.01^{b}$ & $0.03 \pm 0.01^{b}$ & N/D & $\mathrm{N} / \mathrm{S}$ \\
\hline
\end{tabular}

Values with different superscript across columns are different $(\mathrm{P}<0.05)$.

Data are means \pm SEM, $n=8,10$ and 4 for OF-aa, UF-aa and culture media, respectively. OF-aa=oviductal fluid amino acids; UF-aa=uterine fluid amino acids; $\mathrm{K}=\mathrm{KSOM}$; PVA=poly vinyl alcohol; MEM-aa=minimum essential medium amino acids; FCS=fetal calf serum; BSA=bovine serum albumin; N/S=not supplemented; N/D=not detected. 
Table 4. Levels of amino acids in oviductal and uterine fluid samples taken from ovulation and nonovulation sides.

\begin{tabular}{|c|c|c|c|c|}
\hline \multirow{2}{*}{$\begin{array}{l}\text { Amino Acid } \\
\text { (aa) }\end{array}$} & \multicolumn{2}{|c|}{$\underline{\text { OF-aa (mM) }}$} & \multicolumn{2}{|c|}{$\underline{\mathrm{UF}-\mathrm{aa}(\mathrm{mM})}$} \\
\hline & Ovn & N-ovn & Ovn & N-ovn \\
\hline HIS & $0.16 \pm 0.02^{\mathrm{a}}$ & $0.19 \pm 0.02^{\mathrm{a}}$ & $0.69 \pm 0.05^{\mathrm{a}}$ & $0.50 \pm 0.07^{\mathrm{a}}$ \\
\hline THR & $0.81 \pm 0.09^{a}$ & $0.86 \pm 0.09^{\mathrm{a}}$ & $1.96 \pm 0.11^{\mathrm{a}}$ & $1.43 \pm 0.20^{\mathrm{a}}$ \\
\hline $\mathrm{ARG}$ & $0.25 \pm 0.03^{\mathrm{a}}$ & $0.30 \pm 0.03^{\mathrm{a}}$ & $1.69 \pm 0.09^{a}$ & $1.23 \pm 0.16^{b}$ \\
\hline TYR & $0.16 \pm 0.02^{\mathrm{a}}$ & $0.19 \pm 0.01^{\mathrm{a}}$ & $0.73 \pm 0.07^{\mathrm{a}}$ & $0.53 \pm 0.08^{a}$ \\
\hline TRP & $0.15 \pm 0.01^{\mathrm{a}}$ & $0.15 \pm 0.01^{\mathrm{a}}$ & $0.30 \pm 0.01^{\mathrm{a}}$ & $0.26 \pm 0.01^{\mathrm{a}}$ \\
\hline MET & $0.09 \pm 0.01^{\mathrm{a}}$ & $0.11 \pm 0.01^{\mathrm{a}}$ & $0.63 \pm 0.03^{\mathrm{a}}$ & $0.47 \pm 0.06^{\mathrm{a}}$ \\
\hline VAL & $0.46 \pm 0.08^{\mathrm{a}}$ & $0.52 \pm 0.06^{\mathrm{a}}$ & $2.09 \pm 0.17^{\mathrm{a}}$ & $1.61 \pm 0.16^{\mathrm{a}}$ \\
\hline PHE & $0.20 \pm 0.03^{\mathrm{a}}$ & $0.24 \pm 0.02^{\mathrm{a}}$ & $0.79 \pm 0.04^{\mathrm{a}}$ & $0.61 \pm 0.07^{\mathrm{a}}$ \\
\hline ILE & $0.29 \pm 0.02^{\mathrm{a}}$ & $0.33 \pm 0.02^{\mathrm{a}}$ & $0.97 \pm 0.06^{\mathrm{a}}$ & $0.77 \pm 0.09^{\mathrm{a}}$ \\
\hline LEU & $0.44 \pm 0.07^{\mathrm{a}}$ & $0.52 \pm 0.06^{\mathrm{a}}$ & $2.05 \pm 0.13^{\mathrm{a}}$ & $1.58 \pm 0.16^{\mathrm{a}}$ \\
\hline LYS & $0.36 \pm 0.06^{\mathrm{a}}$ & $0.41 \pm 0.04^{\mathrm{a}}$ & $2.06 \pm 0.22^{\mathrm{a}}$ & $1.52 \pm 0.17^{\mathrm{a}}$ \\
\hline CYS & $1.25 \pm 0.32^{\mathrm{a}}$ & $1.46 \pm 0.25^{\mathrm{a}}$ & $0.50 \pm 0.08^{a}$ & $0.61 \pm 0.11^{\mathrm{a}}$ \\
\hline ASP & $0.74 \pm 0.13^{\mathrm{a}}$ & $0.81 \pm 0.08^{\mathrm{a}}$ & $2.05 \pm 0.17^{\mathrm{a}}$ & $1.45 \pm 0.15^{b}$ \\
\hline GLU & $5.25 \pm 0.79^{a}$ & $5.70 \pm 0.60^{\mathrm{a}}$ & $4.53 \pm 0.11^{\mathrm{a}}$ & $3.94 \pm 0.27^{\mathrm{a}}$ \\
\hline ASN & $0.10 \pm 0.01^{\mathrm{a}}$ & $0.11 \pm 0.02^{\mathrm{a}}$ & $0.47 \pm 0.06^{\mathrm{a}}$ & $0.32 \pm 0.09^{\mathrm{a}}$ \\
\hline SER & $0.60 \pm 0.03^{\mathrm{a}}$ & $0.65 \pm 0.04^{\mathrm{a}}$ & $3.07 \pm 0.16^{\mathrm{a}}$ & $2.28 \pm 0.32^{\mathrm{a}}$ \\
\hline GLN & $0.88 \pm 0.22^{\mathrm{a}}$ & $1.00 \pm 0.18^{\mathrm{a}}$ & $2.05 \pm 0.13^{\mathrm{a}}$ & $1.63 \pm 0.20^{\mathrm{a}}$ \\
\hline GLY & $14.83 \pm 2.00^{\mathrm{a}}$ & $13.30 \pm 1.53^{\mathrm{a}}$ & $12.81 \pm 1.71^{\mathrm{a}}$ & $11.18 \pm 1.60^{\mathrm{a}}$ \\
\hline ALA & $3.61 \pm 0.40^{\mathrm{a}}$ & $3.75 \pm 0.58^{\mathrm{a}}$ & $3.48 \pm 0.23^{\mathrm{a}}$ & $3.79 \pm 0.36^{\mathrm{a}}$ \\
\hline PRO & $0.57 \pm 0.12^{\mathrm{a}}$ & $0.57 \pm 0.09^{a}$ & $1.99 \pm 0.11^{\mathrm{a}}$ & $1.77 \pm 0.19^{a}$ \\
\hline CIT & $0.09 \pm 0.02^{\mathrm{a}}$ & $0.10 \pm 0.01^{\mathrm{a}}$ & $0.09 \pm 0.02^{\mathrm{a}}$ & $0.09 \pm 0.02^{\mathrm{a}}$ \\
\hline$\beta-A L A$ & $0.13 \pm 0.00^{\mathrm{a}}$ & $0.14 \pm 0.01^{\mathrm{a}}$ & $0.07 \pm 0.01^{\mathrm{a}}$ & $0.09 \pm 0.01^{\mathrm{a}}$ \\
\hline TAU & $3.61 \pm 0.07^{\mathrm{a}}$ & $3.61 \pm 0.12^{\mathrm{a}}$ & $3.50 \pm 0.84^{\mathrm{a}}$ & $3.36 \pm 0.60^{\mathrm{a}}$ \\
\hline ORN & $0.40 \pm 0.01^{\mathrm{a}}$ & $0.52 \pm 0.01^{\mathrm{a}}$ & $0.16 \pm 0.04^{\mathrm{a}}$ & $0.13 \pm 0.02^{\mathrm{a}}$ \\
\hline
\end{tabular}

Values with different superscript across columns within the same category are different $(\mathrm{P}<0.05)$ Data are means \pm SEM, $\mathrm{n}=5$ and 4 for OF-aa and UF-aa, respectively.

$\mathrm{OF}-\mathrm{aa}=$ oviductal fluid amino acids; UF-aa=uterine fluid amino acids; Ovn=ovulation side; $\mathrm{N}$ ovn=non-ovulation side. 